\title{
Generation of multi-axis Laguerre-Gaussian beams from geometric modes of a hemiconfocal cavity
}

\author{
T.H. Lu • Y.C. Lin · Y.F. Chen · K.F. Huang
}

Received: 12 August 2010 / Revised version: 18 October 2010 / Published online: 27 November 2010

(C) Springer-Verlag 2010

\begin{abstract}
We study the transformation of multi-axis Laguerre-Gaussian beams from optical beams comprising Hermite-Gaussian modes with ray-wave duality. By use of cylindrical lenses, Hermite-Gaussian modes can be transformed into Laguerre-Gaussian modes possessing optical orbital angular momentum. The superposed HermiteGaussian modes localized on geometric trajectories are generated from a degenerate hemiconfocal cavity and transformed into multi-axis Laguerre-Gaussian beams. Experimental results of the structured beams are systematically manipulated and in good agreement with theoretical analyses.
\end{abstract}

\section{Introduction}

In recent years, the study of structured beams has attracted much attention for developing singular optics [1], optical vortices [2-4], and the transfer of optical angular momentum with Bose-Einstein condensates [5-8]. The researches not only pave the way for the applications of microparticle manipulation in optical tweezers [9-11] and encoding of information [12-14] but also visualize the intriguing nature of light [15]. Accordingly, generation of various structured beams plays an important role in realizing the ideas of applications and provides comprehensive insights into wave na-

T.H. Lu (凶)

Department of Physics, National Taiwan Normal University,

88 Tingchou Road, Sec. 4, Taipei 11677, Taiwan

e-mail: thlu@ntnu.edu.tw

Fax: +886-2-29326408

Y.C. Lin · Y.F. Chen · K.F. Huang

Department of Electrophysics, National Chiao Tung University,

Hsinchu, Taiwan ture. A laser cavity was employed to study spatial transverse modes in optical resonators such as $\mathrm{CO}_{2}$ lasers [16, 17], vertical cavity surface emitting lasers [18, 19], and other systems [20-24]. The transverse Hermite-Gaussian modes are the typical modes emitted from most laser cavities. On the other hand, the high-order Laguerre-Gaussian modes related to optical orbital angular momentum can be efficiently generated from diode-pumped solid-state lasers [25, 26].

As is well known, optical spin angular momentum associated with circular polarization was suggested by Poynting [27] and experimentally validated by Beth in 1936 [28]. Optical orbital angular momentum (OAM) related to the spatial distribution was further demonstrated with LaguerreGaussian modes [29]. The compact method to generate Laguerre-Gaussian beams possessing OAM was materialized by cylindrical lenses to transform Hermite-Gaussian beams into Laguerre-Gaussian beams under astigmatism influence $[30,31]$. The Laguerre-Gaussian mode possessing OAM is the general case to study the characteristic of angular momentum of light. Nevertheless, a variety of geometric modes formed by the coherent superposition of HermiteGaussian eigenstates can be generated from degenerate laser resonators [32-34]. By combining the idea of the special coherent waves emitted from a laser cavity and the transformation scheme with cylindrical lenses, intriguing structured beams possessing OAM can be systematically generated.

In this work we present an optical beam generated from a large-Fresnel-number degenerate laser cavity with raywave duality and make use of cylindrical lenses to transform the optical beam into multi-axis Laguerre-Gaussian beams which have circle-bundle structure on the transverse profile. The circle-bundle structured beam represents that the beam with circles uniformly arranged on the circumference results from the superposition of Laguerre-Gaussian modes. We start with the numerical demonstration of the su- 
perposition of Hermite-Gaussian modes which brings about the emergence of localized patterns with ray-wave duality in a degenerate hemiconfocal cavity. The superposed Hermite-Gaussian modes can be converted into the superposed Laguerre-Gaussian mode by use of a mode converter which consists of a pair of cylindrical lenses. Furthermore, the superposed Laguerre-Gaussian modes form the circlebundle structured beams possessing OAM. We employ the case of the degenerate hemiconfocal cavity to clarify the formation of the structured beam. It is the first time that multiaxis Laguerre-Gaussian beams have been generated by the method which combines geometric modes of a laser cavity and the mode converter. Importantly, the experimental results are in good agreement with theoretical analyses and provide striking information for the applications of OAM.

\subsection{Superposition of $1 \mathrm{D}$ and 2D degenerate}

Hermite-Gaussian modes

The wave function of the Hermite-Gaussian mode for the paraxial wave equation in a spherical laser resonator can be written as [35]

$$
\begin{aligned}
\Phi_{n, m, l}^{(\mathrm{HG})}(x, y, z)= & \frac{1}{\sqrt{2^{n+m-1} \pi n ! m !}} \frac{1}{w(z)} H_{n}\left(\frac{\sqrt{2} x}{w(z)}\right) \\
& \times H_{m}\left(\frac{\sqrt{2} y}{w(z)}\right) \exp \left[-\frac{x^{2}+y^{2}}{w(z)^{2}}\right] \\
& \times \exp \left\{-\mathrm{i} k_{n, m, l}\left[1+\frac{x^{2}+y^{2}}{2\left(z^{2}+z_{R}^{2}\right)}\right]\right\} \\
& \times \exp \left[\mathrm{i}(n+m+1) \theta_{\mathrm{G}}(z)\right],
\end{aligned}
$$

where $w(z)=w_{0} \sqrt{1+\left(z / z_{\mathrm{R}}\right)^{2}}, w_{0}$ is the beam radius at the waist, $z_{\mathrm{R}}=\sqrt{L(R-L)}$ is the Rayleigh range, $k_{n, m, l}$ is the wave number, $H_{m}(\cdot)$ is a Hermite polynomial of order $m$, and $\theta_{\mathrm{G}}(z)=\tan ^{-1}\left(z / z_{\mathrm{R}}\right)$ is the Gouy phase. In terms of the cavity length $L$, the wave number $k_{n, m, l}$ is given by $k_{n, m, l} L=\pi\left[l+(n+m+1)\left(\Delta f_{\mathrm{T}} / \Delta f_{\mathrm{L}}\right)\right]$, where $\Delta f_{\mathrm{L}}=c / 2 L$ is the longitudinal mode spacing, $\Delta f_{\mathrm{T}}$ is the transverse mode spacing, $l$ is the longitudinal mode index, and $m$ and $n$ are the transverse mode indices. For an empty resonator consisting of one spherical mirror with radius of curvature $R$ and one plane mirror, the bare ratio between the transverse and the longitudinal mode spacings is given by $\Delta f_{\mathrm{T}} / \Delta f_{\mathrm{L}}=1 / \pi \cos ^{-1}(\sqrt{1-L / R})$. Experimental observations manifest that when the ratio $\Delta f_{\mathrm{T}} / \Delta f_{\mathrm{L}}$ is close to a simple fractional number $P / Q$, the longitudinaltransverse coupling usually brings about frequency locking among different transverse modes with the help of different longitudinal orders [36]. Hence, the three-dimensional coherent optical waves can be expressed as

$$
\begin{aligned}
\Psi_{n_{0}, m_{0}}^{p, q}\left(x, y, z ; \varphi_{0}\right)= & \sum_{k=-M}^{M} C_{M, k} \mathrm{e}^{\mathrm{i} k \varphi_{0}} \\
& \times \Phi_{n_{0}+p k, m_{0}+q k, l_{0}+s k}^{(\mathrm{HG})}(x, y, z)
\end{aligned}
$$

where $C_{M, k}=2^{-M}\left(\begin{array}{c}2 M \\ M+k\end{array}\right)^{1 / 2}$ is the weighting coefficient [37], $\varphi_{0}$ is the relative phase between various HermiteGaussian modes $\Phi_{n, m, l}^{(\mathrm{HG})}(x, y, z)$ at $z=0$, and the indices obey the equation $s+(p+q)(P / Q)=0$ to form a family of frequency-degenerate states. The indices $p$ and $q$ dominate the transverse profile of the coherent optical waves, and the index $s$ matches with the degeneracy in the degenerate cavity, which is decided by $P / Q$. Therefore, $p+q$ needs to be an integral multiple of $Q$. The superposition of degenerate states forms a stationary coherent wave which corresponds to the relation between wave optics and geometric optics in specific initial conditions [32-34]. Fig. 1a depicts a numerical result for the formation of the stationary coherent wave described in e.g. (2) with $z=0, P / Q=1 / 4$, $(p, q)=(0,4),\left(n_{0}, m_{0}\right)=(0,34), M=2$, and $\varphi_{0}=\pi$. The condition $P / Q=1 / 4$ is decided according to the hemiconfocal cavity, and $\left(n_{0}, m_{0}\right)=(0,34)$ and $\varphi_{0}=\pi$ indicate the order of the coherent optical wave and the relative phase of eigenmodes, respectively. The parameter $M=2$ controls the number of eigenmodes and the degree of localization of the coherent optical wave. Importantly, $(p, q)=(0,4)$ represents that there is only one direction of localization because $p=0$ and the localization of four axes can be revealed along the propagation because $q=4$. However, the localization of four axes intersects to form only two bright spots with an interference fringe in the near field. The stationary coherent wave comprises several one-dimensional (1D) HermiteGaussian modes with different orders and leads to the localization in the $y$ direction. Besides, the superposition of two-dimensional (2D) Hermite-Gaussian modes according to the degenerate rule $(p, q)=(0,4)$ leads to the localization in the $y$ direction but not in the $x$ direction. The example shown in Fig. 1b corresponds to Fig. 1a with 2D HermiteGaussian modes of $\left(n_{0}, m_{0}\right)=(3,34)$. The superposition of 1D and 2D Hermite-Gaussian modes according to the same degenerate rule $(p, q)=(0,4)$ results in similar localization, the same localization in one direction but not in another direction.

It is well known that a pure Hermite-Gaussian mode is preserved in free-space propagation. However, superposition of Hermite-Gaussian modes with different transverse orders leads to propagation-variant patterns along the longitudinal direction. Figs. $2 \mathrm{~b}$ and $2 \mathrm{c}$ depict the calculated transverse intensity $\left|\Psi_{n_{0}, m_{0}}^{p, q}\right|^{2}$ described in e.g. (2) at different longitudinal positions corresponding to Figs. 1a and 1b, 
Fig. 1 Numerical results according to (1) with e.g. $(p, q)=(0,4), M=2, \varphi_{0}=\pi$, and $z=0$. (a) Superposition of 1D Hermite-Gaussian modes with the same index $n=0$ and different index $m$.

(b) Superposition of 2D

Hermite-Gaussian modes with the same index $n=3$ and different index $m$

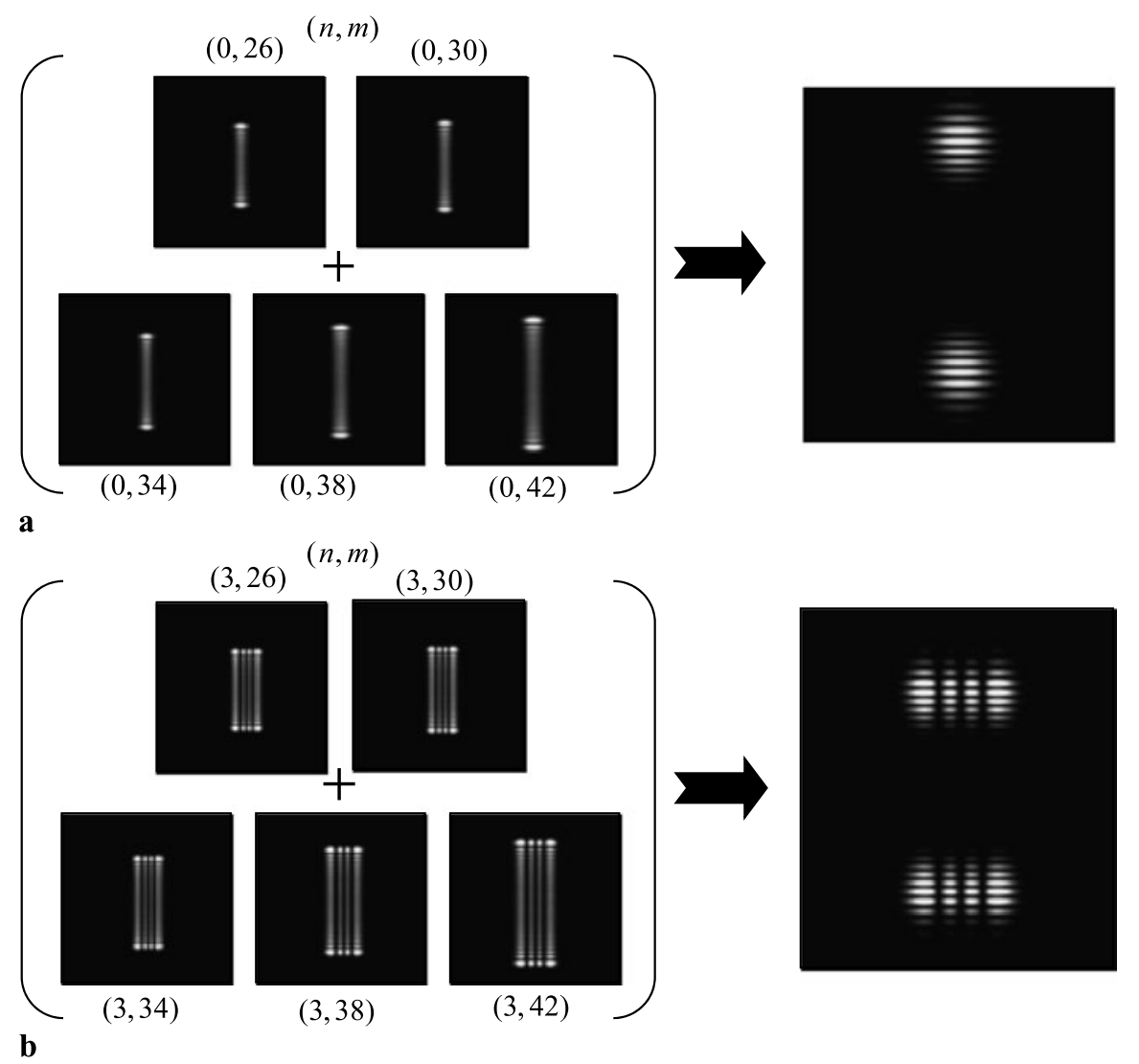

respectively. The different localizations of the transverse intensity under different propagation positions results from the contribution of the Gouy phase of Hermite-Gaussian modes with different orders. For the degenerate superposition of 1D Hermite-Gaussian modes, the coherent wave coincides with the geometrical trajectories shown in Fig. 2a, the so-called $\mathrm{W}$ mode $[32,33]$, in the degenerate cavity with $P / Q=1 / 4$. Obviously, the number of beams traveling under propagation depends on the factor $Q$. On the other hand, the number of axes in the resonator is decided by $Q$. The blue and green arrows indicate the opposite propagation directions of the beam to form the standing waves inside the laser cavity. Note that the degenerate superposition of 1D Hermite-Gaussian modes has a good agreement with the geometrical trajectory but not for 2D Hermite-Gaussian modes shown in Fig. 2c. Consequently, wave representation is a vital approach to explore the deep insights for optical coherent waves [38].

\section{Theoretical analyses for transformation of Hermite-Gaussian modes and superposed Hermite-Gaussian modes}

The relation between Hermite-Gaussian modes and Laguerre-Gaussian modes has been analytically verified and experimentally demonstrated [30, 31, 39]. By use of cylindrical lenses, the Hermite-Gaussian modes with transverse indices $(n, m)$ in rectangular coordinates can be transformed into Laguerre-Gaussian modes with transverse radial index $\tilde{n}$ and azimuthal index $\tilde{m}$ in cylindrical coordinates. The Laguerre-Gaussian mode can be decomposed into a sum of Hermite-Gaussian modes as

$$
\begin{aligned}
\Phi_{\tilde{n}, \tilde{m}, l}^{(\mathrm{LG})}(x, y, z)= & \sum_{u=0}^{2 \tilde{n}+\tilde{m}} \mathrm{e}^{\mathrm{i} u\left(\frac{\pi}{2}\right)} B(\tilde{n}, \tilde{m}, u) \\
& \times \Phi_{2 \tilde{n}+\tilde{m}-u, u, l}^{(\mathrm{HG})}(x, y, z),
\end{aligned}
$$

where

$$
\begin{aligned}
& B(\tilde{n}, \tilde{m}, u) \\
& \quad=\frac{(-1)^{u}}{\sqrt{2^{2 \tilde{n}+\tilde{m}}}} \sum_{v} \frac{(-1)^{v} \sqrt{(\tilde{n}+\tilde{m}) ! \tilde{n} !(2 \tilde{n}+\tilde{m}-u) ! u !}}{v !(u-v) !(\tilde{n}+\tilde{m}-v) !(\tilde{n}+v-u) !} .
\end{aligned}
$$

The relation of the indices between the Hermite-Gaussian modes and the Laguerre-Gaussian modes follows $n=\tilde{n}$ and $m=\tilde{n}+|\tilde{m}|$. The radial index $\tilde{n}$ depends on the index of one direction of Hermite-Gaussian modes, and the azimuthal index $\tilde{m}$ depends on the difference between two indices of Hermite-Gaussian modes. It is worth mentioning that the transformed optical Laguerre-Gaussian modes 
Fig. 2 (a) Diagram of propagation of geometric ray in hemiconfocal cavity.

(b) Numerical patterns of 1D superposed Hermite-Gaussian modes from $z=0$ to far field according to Fig. 1a.

(c) Numerical patterns of 2D superposed Hermite-Gaussian modes from $z=0$ to far field according to Fig. $1 \mathrm{~b}$
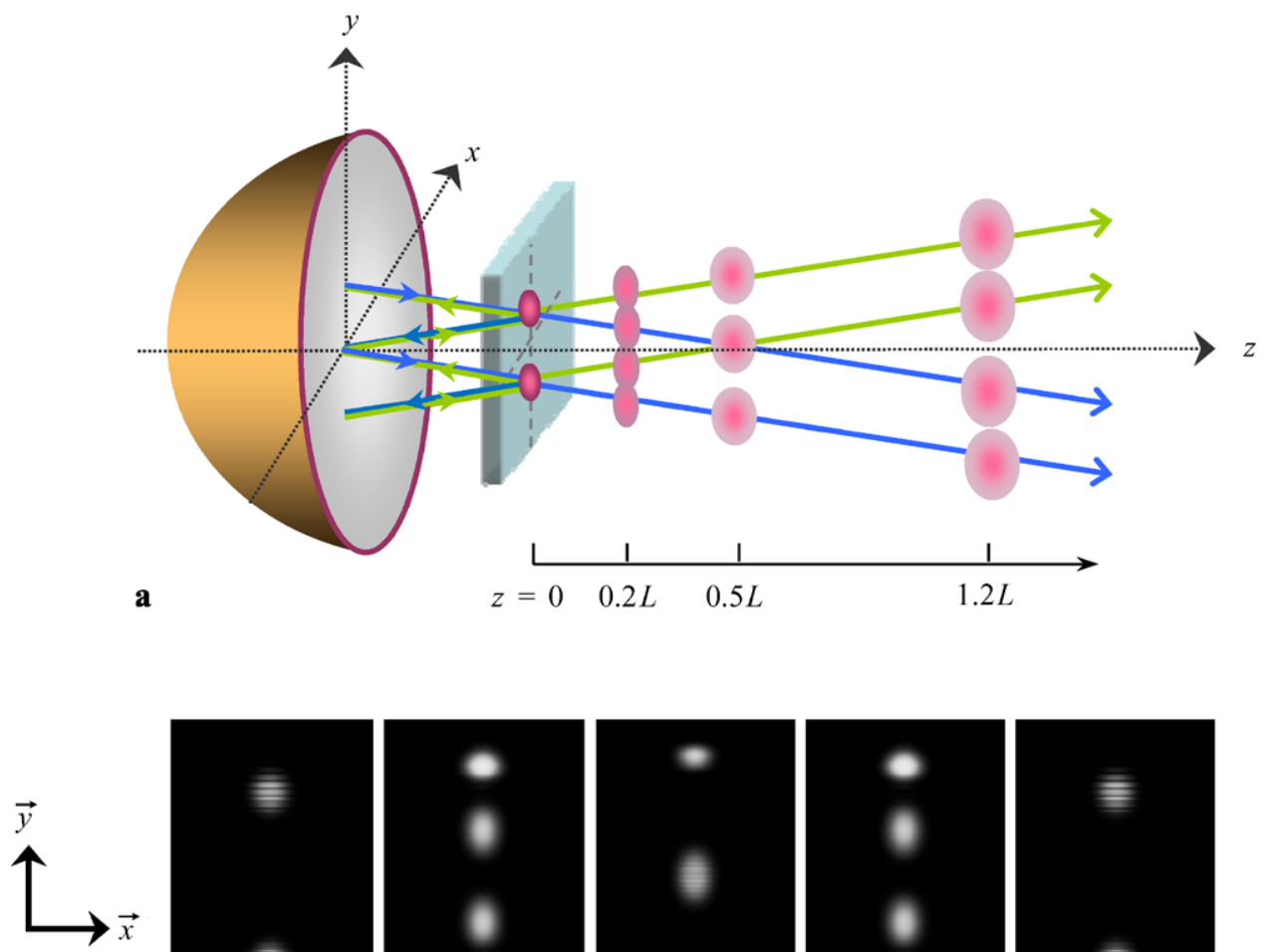

b
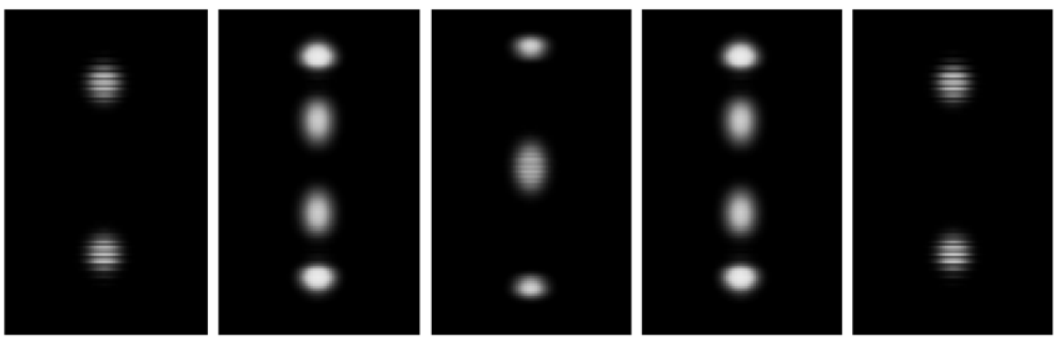

$z=0$

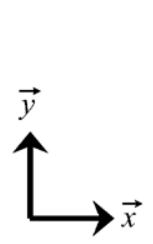

c

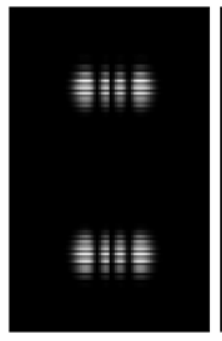

$0.2 L$

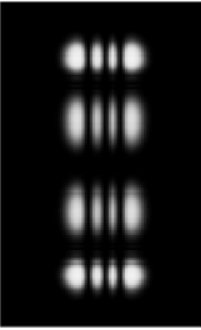

$0.5 L$

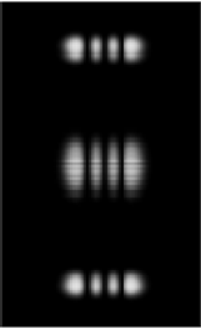

$1.2 L$

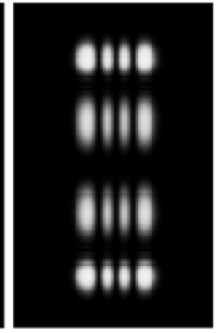

Far field

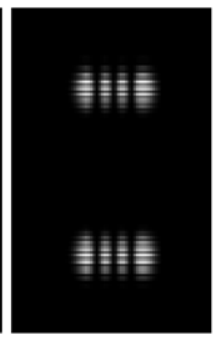

which possess the magnitude of orbital angular momentum $\tilde{m} \hbar$ per photon bring out wide applications for controlling microparticles. In addition to Laguerre-Gaussian modes, there are general astigmatic modes also possessing OAM [40]. Fig. 3 depicts the illustration of transformation between Hermite-Gaussian modes and LaguerreGaussian modes. The left-hand column represents the simulated high-order Hermite-Gaussian modes with transverse indices $(n, m)=(0,4),(1,4)$, and $(2,4)$ at the beam waist of the laser cavity. The right-hand column represents the corresponding simulated Laguerre-Gaussian modes transformed by a pair of cylindrical lenses with transverse indices $(\tilde{n}, \tilde{m})=(0,4),(1,3)$, and $(2,2)$, respectively. From this point of view, we can expand the transformation relation to analyze more complex structured optical waves resulting from coherent superposition of eigenmodes of the optical cavity.
The optical waves with the localized transverse patterns resulting from degenerate superposition of HermiteGaussian modes have been theoretically analyzed and experimentally revealed $[32,33]$. Each composition of the localized coherent wave can be transformed into LaguerreGaussian modes with circular symmetry by a pair of cylindrical lenses mentioned above. Consequently, the superposition of transformed Laguerre-Gaussian modes forms another kind of intriguing pattern we will discuss in the following. From the transformation relation and superposition of coherent modes, the superposed Laguerre-Gaussian modes transformed from superposed Hermite-Gaussian modes can be expressed as

$$
\begin{aligned}
\Psi_{\tilde{n}_{0}, \tilde{m}_{0}}^{\tilde{p}, \tilde{q}}\left(x, y, z ; \varphi_{0}\right)= & \sum_{k=-M}^{M} C_{M, k} \mathrm{e}^{\mathrm{i} k \varphi_{0}} \\
& \times \Phi_{\tilde{n}_{0}+\tilde{p} k, \tilde{m}_{0}+\tilde{q} k, l_{0}+s k}^{(\mathrm{LG})}(x, y, z),
\end{aligned}
$$


Fig. 3 Diagram of mode converter composed of a focusing lens and a pair of cylindrical lenses.

(a)-(c) Numerical Hermite-Gaussian modes before transformation. $\left(\mathbf{a}^{\prime}\right)-\left(\mathbf{c}^{\prime}\right)$ Numerical results after transformation according to (a)-(c), respectively

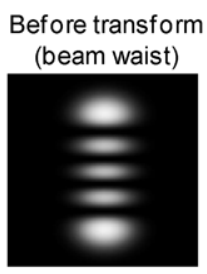

a

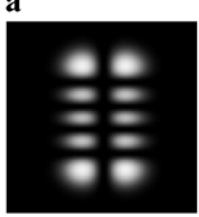

b

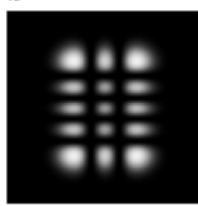

c

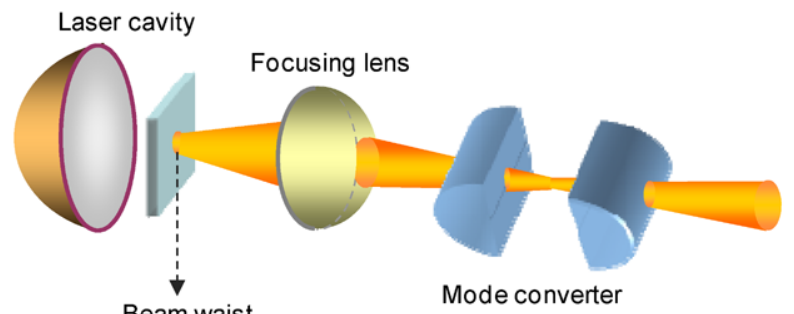

Beam waist

Mode converter
After transform

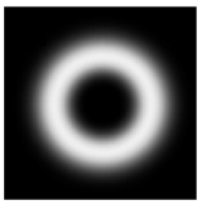

$\mathbf{a}^{\prime}$

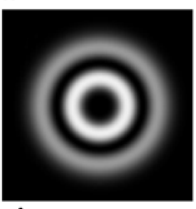

$\mathbf{b}^{\prime}$

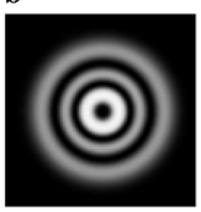

$\mathbf{c}^{\prime}$
Fig. 4 (a) Numerical results of transformed patterns according to Fig. 1a after transformation by mode converter.

(b) Numerical results of transformed patterns according to Fig. 1b after transformation by mode converter

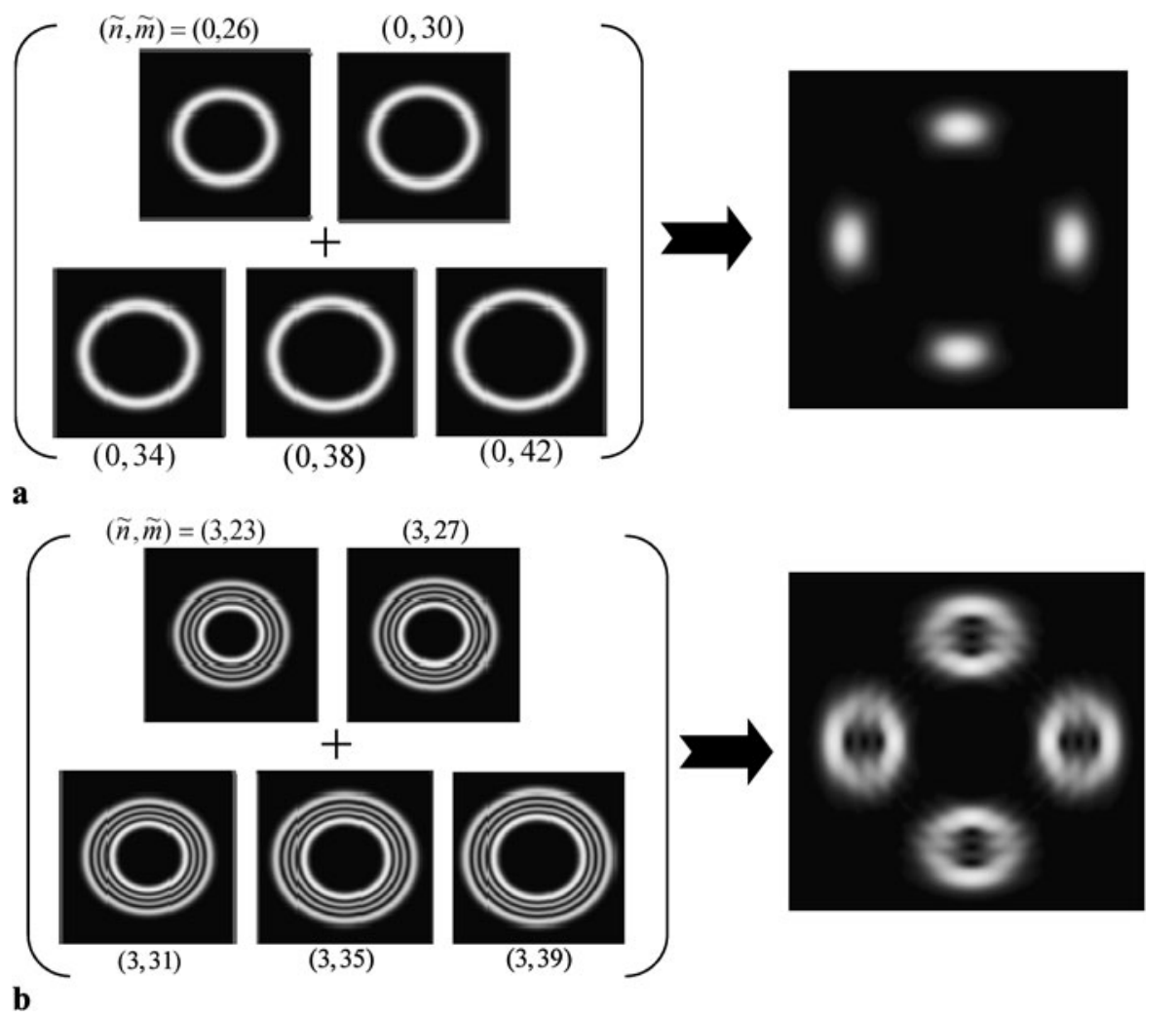

where the indices follow the relations $\tilde{p}=p$ and $\tilde{p}+\tilde{q}=q$. Consequently, we know that the localization of the superposed Laguerre-Gaussian modes is determined by the in$\operatorname{dex} \tilde{p}$ in the radial direction and the index $\tilde{q}$ in the azimuthal direction in the case discussed here. Fig. 4 depicts the numerical results of the superposition of LaguerreGaussian modes which can be transformed with the effect of a pair of cylindrical lenses. In the left-hand part of
Fig. 4a, the single-ring Laguerre-Gaussian modes are the simulated results of individual transformation from the 1D degenerate Hermite-Gaussian modes shown in Fig. 1a with degenerate cavity $P / Q=1 / 4$. The superposition of these transformed Laguerre-Gaussian modes leads to the localization of four azimuthally distributed bright spots shown in the right-hand part of Fig. 4a. Furthermore, the multiring Laguerre-Gaussian modes shown in the left-hand part 
Fig. 5 (a)-(d) Numerical results of superposed Hermite-Gaussian modes according to e.g. (2) with $(p, q)=(0,4), M=2, \varphi_{0}=\pi$, and $\left(n_{0}, m_{0}\right)=(0,36),(12,36)$, $(30,36)$, and $(40,36)$, respectively. $\left(\mathbf{a}^{\prime}\right)-\left(\mathbf{d}^{\prime}\right)$

Numerical results of structured modes transformed from the superposed Hermite-Gaussian modes according to $(\mathbf{a})-(\mathbf{d})$
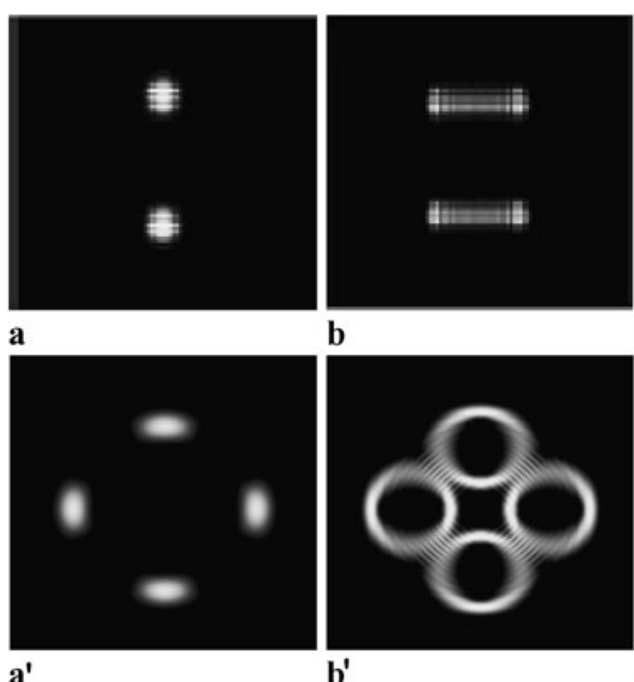

b

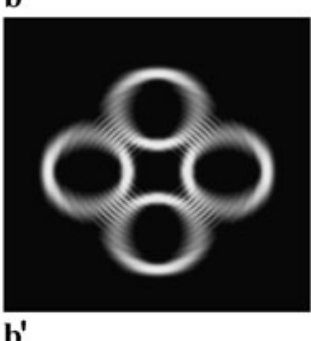

$\mathbf{b}^{\prime}$
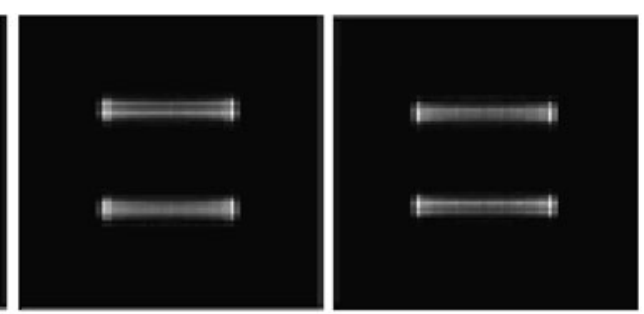

c

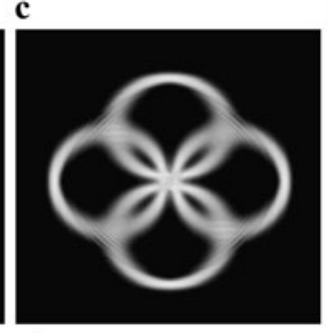

d

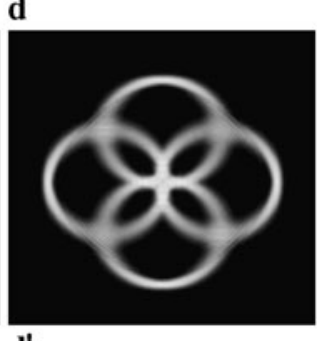

c'

d'

\section{Realization of transformation of superposed Hermite-Gaussian modes}

mation from the 2D degenerate Hermite-Gaussian modes shown in Fig. $1 \mathrm{~b}$ with degenerate cavity $P / Q=1 / 4$. Different from the four bright spots, the superposition of the multi-ring Laguerre-Gaussian modes forms the intriguing transverse pattern with azimuthally distributed circular bundles. The structured mode with azimuthally distributed circles resulted from the superposition of Laguerre-Gaussian modes with the same radial index $\tilde{n}$ but different azimuthal index $\tilde{m}$, so the diameter of the circles is decided by the structure of the Laguerre-Gaussian mode with the average azimuthal index $\tilde{m}$ and the radial index $\tilde{n}$. The diameter of the circle is equal to the distance between the inner ring and the outer ring along the radial direction of the LaguerreGaussian mode with the average azimuthal index $\tilde{m}$ and is proportional to the radial index $\tilde{n}$ of Laguerre-Gaussian modes in different cases. The transformed structured modes depend on the type of 1D or 2D superposed HermiteGaussian modes. Therefore, we show the representative numerical results in Fig. 5 to reveal the effective parameter for the formation of different structured modes through the transformation by cylindrical lenses. Figures $5 \mathrm{a}-5 \mathrm{~d}$ depict the numerical far-field patterns for the formation of superposed Hermite-Gaussian modes described in e.g. (2) with $P / Q=1 / 4,(p, q)=(0,4), m_{0}=36, M=2, \varphi_{0}=\pi$, and several different $n_{0}$. The corresponding simulated results of the transformation by a pair of cylindrical lenses are shown in Figs. $5 \mathrm{a}^{\prime}-5 \mathrm{~d}^{\prime}$. As the order of superposed HermiteGaussian modes gets higher, the diameter of the circles also gets larger so that they cross each other. Each numerical result of Figs. $5 \mathrm{a}^{\prime}-5 \mathrm{~d}^{\prime}$ represents that there are four LaguerreGaussian modes along four axes to clarify the multi-axis Laguerre-Gaussian beam.
The large-Fresnel-number degenerate cavity has been employed to generate miscellaneous optical coherent waves localized on geometric trajectories and classical parametric surfaces [33, 36, 41]. Moreover, the optical coherent waves emitted from the large-Fresnel-number degenerate cavity can be used to study the generation of large optical angular momentum [42]. Angular momentum transferred from a Laguerre-Gaussian beam is the first demonstration of optical angular momentum. As is well known, a LaguerreGaussian beam with orbital angular momentum can be efficiently generated from a Hermite-Gaussian beam by use of a pair of cylindrical lenses [30, 31]. Consequently, it is generally interesting for developing the structured beams with angular momentum from superposed Hermite-Gaussian modes. To realize the superposed Hermite-Gaussian modes of the large-Fresnel-number degenerate cavity, the experimental laser cavity was composed of a spherical mirror and a gain medium shown in Fig. 2a. The spherical mirror was a $10 \mathrm{~mm}$ radius-of-curvature concave mirror with antireflection coating at the pumping wavelength on the entrance face $(R<0.2 \%)$, high reflection coating at the lasing wavelength $(R>99.8 \%)$, and high-transmission coating at the pumping wavelength on the other surface $(T>95 \%)$. The gain medium was an $a$-cut 2.0 at $\% \mathrm{Nd}: \mathrm{YVO}_{4}$ crystal with a length of $2 \mathrm{~mm}$ and a cross section of $3 \times 3 \mathrm{~mm}^{2}$. One planar surface of the laser crystal was coated for antireflection at the pumping and lasing wavelengths; the other surface was coated to be an output coupler with a reflectivity of $99 \%$. The pump source was an 809 -nm fiber-coupled laser diode with a core diameter of $100 \mu \mathrm{m}$ and a maximum output power of $1 \mathrm{~W}$. A coupling lens was used to focus the pump beam to be approximately $25 \mu \mathrm{m}$ in the laser crystal. 
Fig. 6 (a)-(d) Experimental results of superposed Hermite-Gaussian modes with ray-wave duality in hemiconfocal resonator with off-axis magnitude $140 \mu \mathrm{m}$ in the $y$ direction and the magnitude $0 \mu \mathrm{m}, 90 \mu \mathrm{m}$, $130 \mu \mathrm{m}$, and $170 \mu \mathrm{m}$ in the $x$ direction, respectively. $\left(\mathbf{a}^{\prime}\right)-\left(\mathbf{d}^{\prime}\right)$ Experimental results of structured beams transformed from the superposed Hermite-Gaussian modes shown in (a) $-(\mathbf{d})$

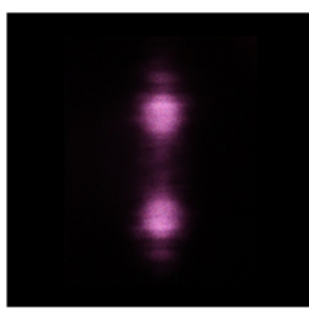

a

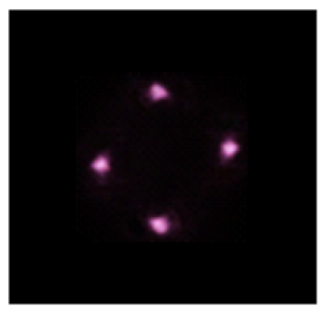

$a^{\prime}$

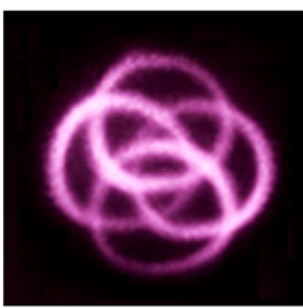

a

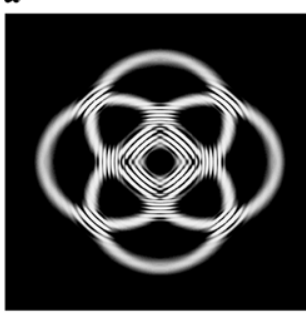

$a^{\prime}$

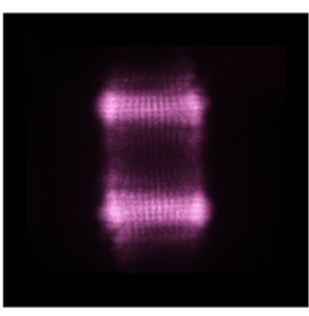

b

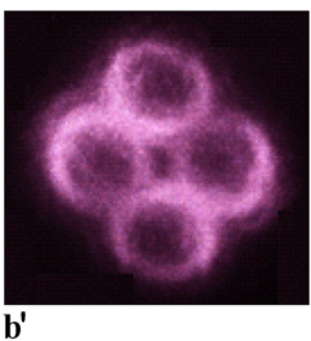

$\mathbf{b}^{\prime}$

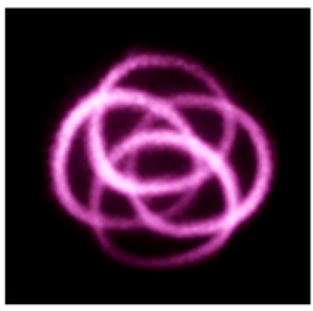

b

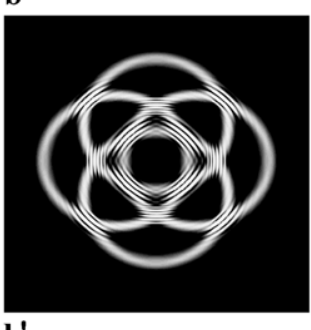

$b^{\prime}$

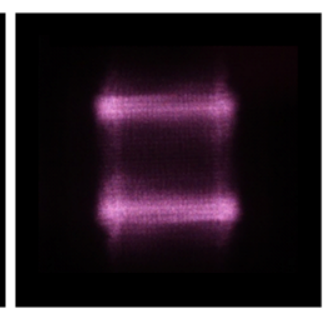

c

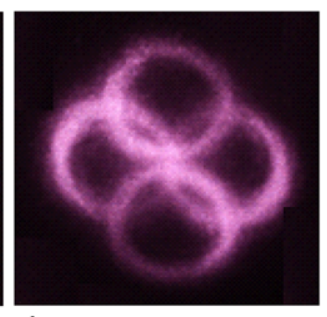

$c^{\prime}$

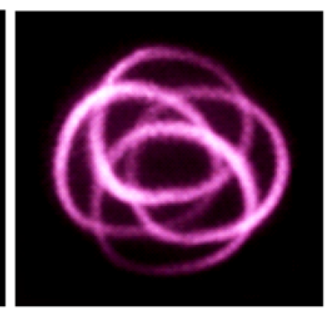

c

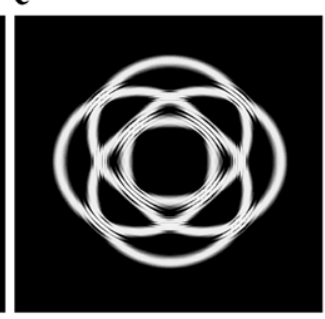

$\mathbf{c}^{\prime}$
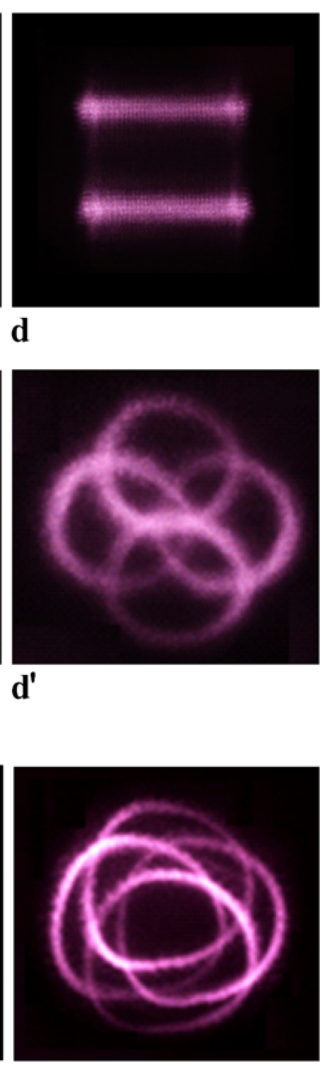

d

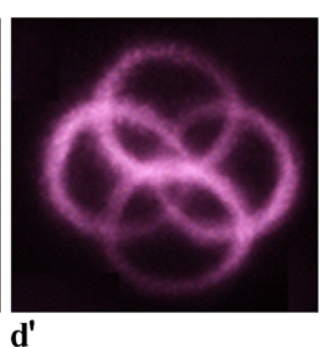

d

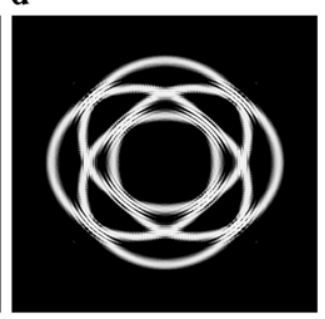

$d^{\prime}$
Figs. 6a-6d depict the experimental far-field results of the superposed 1D and 2D Hermite-Gaussian modes related to the degenerate cavity $P / Q=1 / 4$ under the condition of different $x$ off-axis and the same $y$ off-axis. The propagation behavior of these localized coherent waves is confirmed as the theoretical result shown in Fig. 2, and the superposed Hermite-Gaussian modes possess no optical angular momentum. By use of a pair of cylindrical lenses with focal length of $25 \mathrm{~mm}$ and a focusing lens, the input beam emitted from the laser cavity is focused to the mid point of the two cylindrical lenses shown in Fig. 3, and the superposed Hermite-Gaussian modes can be transformed to the multiaxis Laguerre-Gaussian beams shown in Figs. 6a'-6d'. Importantly, the experimental patterns are in good agreement with the feature of the theoretical results shown in Fig. 5. The larger off-axis conditions of the experimental results for the transformed structured beams are shown in Figs. 7a-7d. The imperfect patterns under the extreme off-axis condition are caused by the aberration of the focusing lens. Nevertheless, the results are still in good agreement with the theoretical results shown in Figs. $7 \mathrm{a}^{\prime}-7 \mathrm{~d}^{\prime}$.

The Laguerre-Gaussian mode which possesses the magnitude of OAM $\tilde{m} \hbar$ per photon only depends on the azimuthal index $\tilde{m}$ [29]. Hence, we can derive that the OAM of the multi-axis Laguerre-Gaussian beams which are the superposition of Laguerre-Gaussian modes only depends on the average azimuthal index $\tilde{m}_{0}$. For the structured beams in Figs. $6 \mathrm{a}^{\prime}-6 \mathrm{~d}^{\prime}$, the OAM per photon can be approximately calculated at around $36 \hbar, 24 \hbar, 6 \hbar$, and $-4 \hbar$ according to the numerical results in Figs. $5 \mathrm{a}^{\prime}-5 \mathrm{~d}^{\prime}$. In addition, the magnitude of OAM per photon of the structured beams shown in Figs. $7 \mathrm{a}-7 \mathrm{~d}$ can be approximately calculated at around $-22 \hbar,-42 \hbar,-82 \hbar$, and $-117 \hbar$ according to the numerical results in Figs. $7 a^{\prime}-7 d^{\prime}$. The positive or negative OAM represents whether the rotation around the optical axis is counterclockwise or clockwise. The results reveal that the mag- 
Mode converter

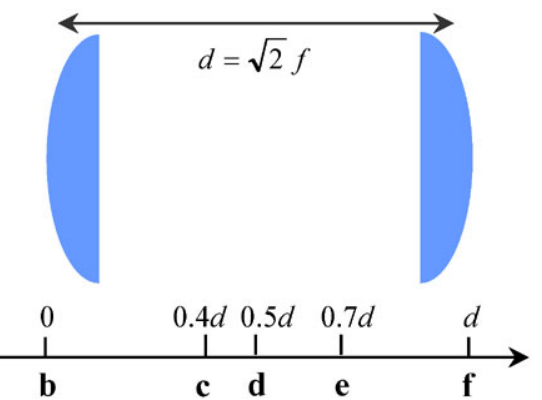

a
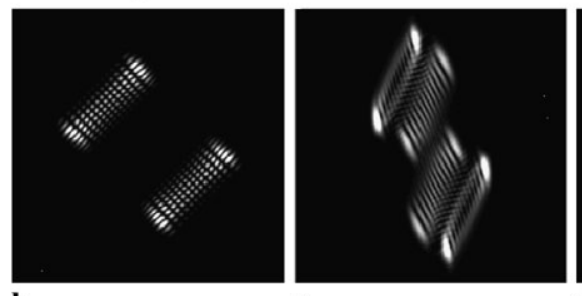

c
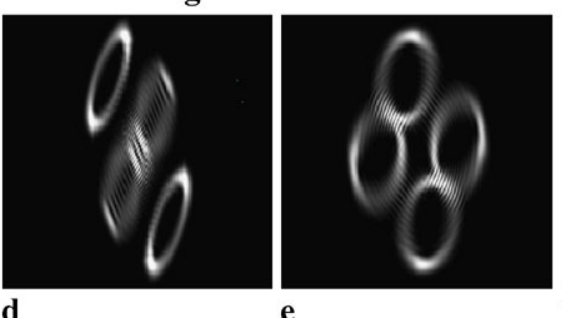

e

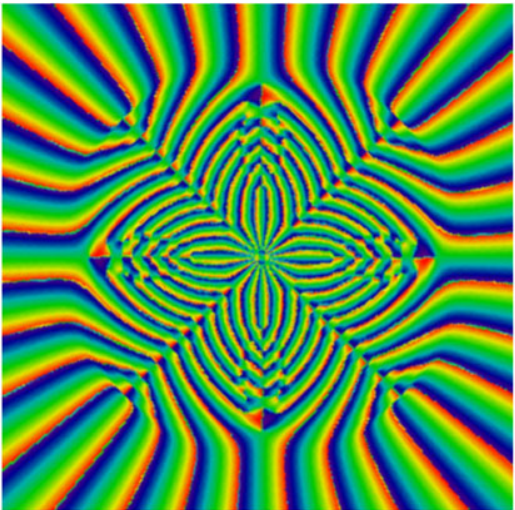

g

Fig. 8 Diagram of the distance between two cylindrical lenses. (b)-(f) Propagation-dependent numerical results with $\left(n_{0}, m_{0}\right)=(12,36)$ at different positions between two cylindrical lenses marked in (a). (g) Phase distribution of the numerical circle-bundle structured beam shown in (f)

nitude of OAM per photon is proportional to the difference between the two indices $\left(n_{0}, m_{0}\right)$ of the input superposed Hermite-Gaussian mode.

Finally, it is worth mentioning that the three-dimensional structure of the circle-bundle beams is propagation dependent because of the Gouy phase and the additional phase of the mode converter. Figs. $8 \mathrm{~b}-8 \mathrm{f}$ depict the numerical results of the tomographic transverse patterns under propagation. Note that the distance between the two cylindrical lenses should be set as $\sqrt{2} f$ to complete the transformation [31]. The numerical results are based on the ABCD law to estimate the astigmatic effect from the mode converter. Moreover, the phase distribution of the numerical circle-bundle structured beam shown in Fig. 8f is revealed in Fig. 8g.

\section{Conclusion}

In summary, we have successfully employed the representation of the superposed Hermite-Gaussian modes and the function of the mode converter to expound the formation of multi-axis Laguerre-Gaussian beams possessing OAM. The experimental structured beams are robust and can be facilely manipulated by controlling the superposed Hermite-Gaussian modes generated from the large-Fresnelnumber degenerate cavity. Moreover, the experimental results are in good agreement with the theoretical results. Since the intriguing structured beams are elegantly generated with considerable OAM, the present findings may provide some useful insights into various optical applications.
Acknowledgement The authors thank the National Science Council for their financial support of this research under Contract No. NSC-982112-M-003-008-MY3.

\section{References}

1. M.S. Soskin, M.V. Vasnetsov, in Progress in Optics, vol. 42, ed. by E. Wolf (ed.) (Elsevier, Amsterdam, 2001), pp. 219-276

2. D. Rozas, C.T. Law, G.A. Swartzlander, J. Opt. Soc. Am. B 14, 3054 (1997)

3. Y. Izdebskaya, T. Fadeyeva, V. Shvedov, A. Volyar, Opt. Lett. 31, $2523(2006)$

4. S.M. Baumann, D.M. Kalb, L.H. MacMillan, E.J. Galvez, Opt. Express 17, 9818 (2009)

5. K.T. Kapale, J.P. Dowling, Phys. Rev. Lett. 95, 173601 (2005)

6. M.F. Andersen, C. Ryu, P. Cladé, V. Natarajan, A. Vaziri, K. Helmerson, W.D. Phillips, Phys. Rev. Lett. 97, 170406 (2006)

7. T.P. Simula, N. Nygaard, S.X. Hu, L.A. Collins, B.I. Schneider, K. Mølmer, Phys. Rev. A 77, 015401 (2008)

8. K.C. Wright, L.S. Leslie, N.P. Bigelow, Phys. Rev. A 77, 041601 (2008)

9. K.T. Gahagan, G.A. Swartzlander Jr., Opt. Lett. 21, 827 (1996)

10. N.B. Simpson, K. Dholakia, L. Allen, M.J. Padgett, Opt. Lett. 22, 52 (1997)

11. H. Adachi, S. Akahoshi, K. Miyakawa, Phys. Rev. A 75, 063409 (2007)

12. J.C. Garcia-Escartin, P. Chamorro-Posada, Phys. Rev. A 78, $062320(2008)$

13. E. Nagali, F. Sciarrino, F. Martini, L. Marrucci, B. Piccirillo, E. Karimi, E. Santamato, Phys. Rev. Lett. 103, 013601 (2009)

14. L. Chen, W. She, New J. Phys. 11, 103002 (2009)

15. D.L. Andrews, Structured Light and its Applications: An Introduction to Phase-Structured Beams and Nanoscale Optical Forces (Academic Press/Elsevier, Burlington, 2008) 
16. D. Dangoisse, D. Hennequin, C. Lepers, E. Louvergneaux, P. Glorieux, Phys. Rev. A 46, 5955 (1992)

17. E. Louvergneaux, D. Hennequin, D. Dangoisse, P. Glorieux, Phys. Rev. A 53, 4435 (1996)

18. G. D’Alessandro, F. Papoff, E. Louvergneaux, P. Glorieux, Phys. Rev. E 69, 066212 (2004)

19. X. Hachair, S. Barbay, T. Elsass, I. Sagnes, R. Kuszelewicz, Opt. Express 16, 9519 (2008)

20. Ch. Tamm, Phys. Rev. A 38, 5960 (1988)

21. M. Brambilla, F. Battipede, L.A. Lugiato, V. Penna, F. Prati, C. Tamm, C.O. Weiss, Phys. Rev. A 43, 5090 (1991)

22. M.C. Cross, P.C. Hohenberg, Rev. Mod. Phys. 65, 851 (1993)

23. A.B. Coates, C.O. Weiss, C. Green, E.J. D’Angelo, J.R. Tredicce, M. Brambilla, M. Cattaneo, L.A. Lugiato, R. Pirovano, F. Prati, A.J. Kent, G.-L. Oppo, Phys. Rev. A 49, 1452 (1994)

24. S.J.M. Habraken, G. Nienhuis, Phys. Rev. A 75, 033819 (2007)

25. A.A. Ishaaya, N. Davidson, A.A. Friesem, Opt. Express 13, 4952 (2005)

26. M.P. Thirugnanasambandam, Yu. Senatsky, K. Ueda, Laser Phys. Lett. 7, 637-643 (2010)

27. J.H. Poynting, Proc. R. Soc. Lond. Ser. A 82, 560 (1909)

28. R.A. Beth, Phys. Rev. 50, 115 (1936)

29. L. Allen, M.W. Beijersbergen, R.J.C. Spreeuw, J.P. Woerdman, Phys. Rev. A 45, 8185 (1992)
30. E. Abramochkin, V. Volostnikov, Opt. Commun. 83, 123 (1991)

31. M.W. Beijersbergen, L. Allen, H.E.L.O. van der Veen, J.P. Woerdman, Opt. Commun. 96, 123 (1993)

32. J. Dingjan, M.P. van Exter, J.P. Woerdman, Opt. Commun. 188, 345 (2001)

33. Y.F. Chen, C.H. Jiang, Y.P. Lan, K.F. Huang, Phys. Rev. A 69, 053807 (2004)

34. J. Visser, N.J. Zelders, G. Nienhuis, J. Opt. Soc. Am. A 22, 1559 (2005)

35. A.E. Siegman, Lasers (University Science Books, Mill Valley, 1986)

36. Y.F. Chen, T.H. Lu, K.W. Su, K.F. Huang, Phys. Rev. Lett. 96, 213902 (2006)

37. M.S. Kumar, B. Dutta-Roy, J. Phys. A 41, 075306 (2008)

38. G. Nienhuis, L. Allen, Phys. Rev. A 48, 656 (1993)

39. S. Danakas, P.K. Aravind, Phys. Rev. A 45, 1973 (1992)

40. J. Visser, G. Nienhuis, Phys. Rev. A 70, 013809 (2004)

41. T.H. Lu, Y.C. Lin, Y.F. Chen, K.F. Huang, Phys. Rev. Lett. 101, $233901(2008)$

42. T.H. Lu, Y.C. Lin, Y.F. Chen, K.F. Huang, Opt. Express 17, 3007 (2009) 